Edith Cowan University

Research Online

Research outputs 2014 to 2021

$12-20-2017$

\title{
The interaction between encapsulated Gd203 particles and polymeric matrix: The mechanism of fracture and X-ray attenuation properties
}

\author{
Ly B. T. La \\ Edith Cowan University \\ Christopher Leatherday \\ Peng Qin \\ Edith Cowan University \\ Yee-Kwong Leong \\ Kevin Hayward \\ Edith Cowan University
}

See next page for additional authors

Follow this and additional works at: https://ro.ecu.edu.au/ecuworkspost2013

Part of the Engineering Commons

\footnotetext{
This is an Author's Accepted Manuscript of:

La, L. B. T., Leatherday, C., Qin, P., Leong, Y.-K., Hayward, K. J., Jiang, B., \& Zhang, L.-C. (2017). The interaction between encapsulated Gd2O3 particles and polymeric matrix: The mechanism of fracture and X-ray attenuation properties. Colloids and Surfaces A: Physicochemical and Engineering Aspects, 535(Supplement C), 175-183. https://doi.org/10.1016/j.colsurfa.2017.09.038

This Journal Article is posted at Research Online.

https://ro.ecu.edu.au/ecuworkspost2013/3593
} 


\section{Authors}

Ly B. T. La, Christopher Leatherday, Peng Qin, Yee-Kwong Leong, Kevin Hayward, Bin Jiang, and Lai-Chang Zhang

This journal article is available at Research Online: https://ro.ecu.edu.au/ecuworkspost2013/3593 


\section{CHAPTER 7: THE INTERACTION BETWEEN ENCAPSULATED Gd $\mathrm{G}_{2}$ PARTICLES AND POLYMERIC MATRIX: THE MECHANISM OF FRACTURE AND X-RAY ATTENUATION PROPERTIES}

\subsection{Introduction}

Cured epoxy shows favourable engineering properties such as high elastic modulus and good thermal, and dimensional stabilities compared to other thermosetting materials. However, it has poor resistance to crack initiation, propagation and poor interior impact strength due to its highly cross-linked structure[81,82,217]. The addition of the correct amount of an appropriate rigid metal or ceramic filler at not only improves the fracture toughness properties for practical applications, but also extends to the multifunctional applications of the cured epoxy. For example, the fillers of titanium compounds and clay glass fibers accounted for the greatest improvement of mechanical properties of the polymer in the context of its use as an automobile component[5,6,218].Additionally, calcium carbonate $\left(\mathrm{CaCO}_{3}\right)$, silica $\left(\mathrm{SiO}_{2}\right)$, and aluminum oxide $\left(\mathrm{Al}_{2} \mathrm{O}_{3}\right)$ fillers increased the dielectric constant and thermal conductivity of the epoxy, making them suitable for use as capacitors in the electronic packing industry [14,16]. Gadolinium oxide $\left(\mathrm{Gd}_{2} \mathrm{O}_{3}\right)$ filler was also reported to form a non-toxic or "green" radiation protective epoxy composite[7,69]. The first report on $\mathrm{Gd}_{2} \mathrm{O}_{3}$-epoxy (Gd-epoxy) nanocomposite specified a maximum particle volume fraction of 0.017 for fracture toughness improvement [7]. At this volume fraction, a $1 \mathrm{~cm}$ thickness $\left(1.258 \mathrm{~g} / \mathrm{cm}^{3}\right)$ could attenuate $50-70 \%$ ofX-rays in the range of $40-100 \mathrm{kVp}$ [7]. However, nanosize $\mathrm{Gd}\left(\mathrm{Gd}_{2} \mathrm{O}_{3}\right)$ fillers formed bulky clusters greater than $5 \mu \mathrm{m}$ in diameter in the epoxy resin [7]. This research suggested that the low loading and ineffective dispersion of the particles in the polymer matrix hindered both the mechanical property improvement and the radiation protective performance.

Particulate hybrid composites are expected to inherit distinguishing properties of both the polymer matrix and reinforcing fillers; however, many other parameters also come into play in determining composite properties, especially toughness and functionality. For agiven filler material, particulate loading significantly influences the toughness and functional properties of a polymer composite. The size and shape of the particles, and the interfacial bonding between particle and matrix are the most important parameters defining the dispersion status of the particles in the cured polymer matrix. Normally, the plane strain fracture toughness 
$\left(\mathrm{K}_{\mathrm{IC}}\right)$ and critical strain energy release rate $\left(\mathrm{G}_{\mathrm{IC}}\right)$, which characterize the mechanical properties of a composite, increase initially with particulate concentration to a plateau value and then they decrease with further increases in filler concentration [217,219,220]. The functional properties inherited from the rigid filler, such as radiation attenuation for $\mathrm{Gd}_{2} \mathrm{O}_{3}$ or electric insulation for $\mathrm{CaCO}_{3}$, display a sharp and then steady increase with an increasing filler concentration $[69,220]$. Several reports also indicate that composite properties such as tearing strain and toughness are determined by particle quantity, interactions, size, and the particle-matrix bonding strength [220,221].

Nano-scale primary particles are ideal candidates for a particulate composite product if they can be dispersed uniformly in the polymeric phase. Unfortunately, agglomeration and coprecipitation of fine particles are often observed in a particulate composite, resulting in low wettability and weak bonding between an inorganic filler andan organic matrix because of the chemical incompatibility of the interface[220]. For a given volume fraction, smaller particles represent a larger interfacial area, which would give rise to a greater tendency of particle agglomeration[220,221]. Appropriate surface modification of inorganic particles can prevent aggregation and can strengthen the particle/matrix adhesion, which results in a more uniform dispersion. At the same time, the uniformly dispersed particles would act as crack stoppers, thereby preventing crack growth of propagation. This should also lead to an increase in the particle loading concentration.

This workinvolved dispersing different quantities of synthesized and surface modified nano $\mathrm{Gd}_{2} \mathrm{O}_{3}$ particles in epoxy by ultrasonic means. Thismethod can ease the ex-situ process because it doesn't require adding volatile solvents. The mechanical properties and X-ray protection of $\mathrm{Gd}_{2} \mathrm{O}_{3}$ particulate compositeswere characterized and analyzed with the objective of determining the synthesized conditionsfor optimum properties of this material.It is hoped that the inorganic filler can not only add more multifunctionality to epoxy matrices, but also increase the fracture toughness of neat epoxies without sacrificing their base properties. The performances of the resultant compositeswere compared to other similar results reported elsewhere.

\subsection{Materials and method}

\subsubsection{Materials}

The gadolinium oxide $\left(\mathrm{Gd}_{2} \mathrm{O}_{3}, 99.99 \%\right.$ pure, denoted as $\left.\mathrm{Gd}\right)$ with a diameter of less than $30 \mu \mathrm{m}$ was supplied by Sun Chemical Technology Co. Cured epoxy matrix was produced by 
blending epoxy resins, bisphenol-A-(epichlorhydrin) (average molecular weight $\leq 700$ ) and triethylenetetramine hardener, which were purchased from Struers, US. Anionic surfactant, sodium dodecyl sulfate (SDS) with 99\% purity, Sodium hydroxide pellets $(\mathrm{NaOH})$ and a $40 \%$ solution of nitric acid $\left(\mathrm{HNO}_{3}\right)$ were provided by Sigma Aldrich. Sodium chloride crystals $(\mathrm{NaCl}, 99 \%)$ were purchased from Alfa Aesar.

\subsubsection{Method}

7.2.2.1. Fabrication of Gdnanoparticle core with organic shell and particulate nanocomposite development.

Sodium chloride crystals mixed with the $\mathrm{Gd}_{2} \mathrm{O}_{3}$ powder at a mass ratio of 1.5: 1 were grinded in a high-energy ball mill (A SPEX $8000 \mathrm{M}$ ) at room temperature and pressure for 1.5 hours. The milled mixture was washed using deionized water and enough $\mathrm{NaOH}$ pellets were added to increase the $\mathrm{pH}$ to around 11 (near the isoelectric point of the oxide), to enhance the sedimentation rate of the aggregated $\mathrm{Gd}_{2} \mathrm{O}_{3}$ particles. This washing process was then repeated several times with DI water until the conductivity of the decanted solution was less than $0.5 \mathrm{mS} / \mathrm{cm}$. The cleaned suspension was concentrated by centrifugation to obtain a thickened suspension with a particle volume fraction $\left(\phi_{s}\right)$ of 0.2 using a Sigma 2-6 centrifuge (Germany). The $\mathrm{pH}$ of the thickened suspension was adjusted to around 7 with a $0.7 \mathrm{M} \mathrm{HNO}_{3}$ solution causing a large reduction in the suspension viscosity. Anionic surfactant, $0.1 \mathrm{dwb} \%$ (dry weight basis percentage) of sodium dodecyl sulfate (SDS), was dissolved in this low slurry and the resultant suspension was then dispersed with a Branson sonifier operated at $70 \%$ amplitude. The treated suspension was further heat-treated and dried at $60{ }^{\circ} \mathrm{C}$ in an oven for 7 days. The resultant $\mathrm{Gd}_{2} \mathrm{O}_{3}$ nanoparticles had been encapsulated with an organic surfactant layer (modified Gd), and were dispersed in epoxy resin using the sonifer set at $50 \%$ amplitude. After that, a cross linking agent was added, and the resultant suspension was degassed, placed in a home-made mould, and left for about 12 hours at ambient conditions for curing or hardening to occur. Cured Gd/epoxy nanocomposite at different solid volume fractions $\left(\phi_{s}\right)$ of $0.0,0.04,0.06,0.08,0.1,0.12$, and 0.14 were prepared.

\subsubsection{Structure and chemical characterization}

The particle size distribution of modified Gd was analyzed by laser diffraction (Malvern Mastersizer Microplus Particle Size Analyzer). A Zeiss 1555 VPSEM scanning electron microscope (SEM) was also employed to investigate the morphology of synthesized $\mathrm{Gd}_{2} \mathrm{O}_{3}$ nanoparticles and to observe the fractograph of particulate composite. This information would 
help evaluate the dispersion status of the particles in the epoxy matrix. The presence of the outer organic surfactant layer around the $\mathrm{Gd}_{2} \mathrm{O}_{3}$ particles was characterized by a Spectrum One Fourier transform infrared spectrometer (FTIRspectrometers) from Perkin Elmer.

\subsubsection{Mechanical properties}

An Instron 5982 universal testing machine and its extensometer were employed to evaluate the mechanical properties of the $\mathrm{Gd}_{2} \mathrm{O}_{3}$ particulate composite at different solid volume fractions $\left(\phi_{s}\right)$ from 0 to 0.14 . Five dumb-bell specimens, based on the ASTM standard D63802a specification of a gauge length of $50 \mathrm{~mm}$ and constant thickness of $5 \pm 1 \mathrm{~mm}$, were prepared for each composite composition. The crosshead rate of $0.5 \mathrm{~mm} / \mathrm{min}$ was used for tensile mechanical testing. The elastic deformation of composite under load (young's modulus) was estimated from the gradient of the stress-strain curve. All the mechanical properties reported in this work were averaged from at least four individual tests.

Five compact tension specimens were also prepared for each composite composition to determine their fracture toughness. Again, a crosshead rate of $0.5 \mathrm{~mm} / \mathrm{min}$ was employed. The fracture toughness characterizes the material's resistance to brittle fracture in the presence of a crack. Two important parameters were calculated from the tests, i.e., the plane strain critical stress concentration factor $\left(\mathrm{K}_{\mathrm{IC}}\right)$ and the critical strain energy release rate $\left(\mathrm{G}_{\mathrm{IC}}\right)$, according to ASTM D 5045-99. The compact tension specimen configuration is shown inFigure 7.1.A sharp crack was made by a razor tapping method which was followed by a pre-notch crack cut with a band saw.The razor crack length was in the range of $0.45<\mathrm{a} / \mathrm{W}<$ 0.55 . The $\mathrm{K}_{\mathrm{IC}}$ is given by:

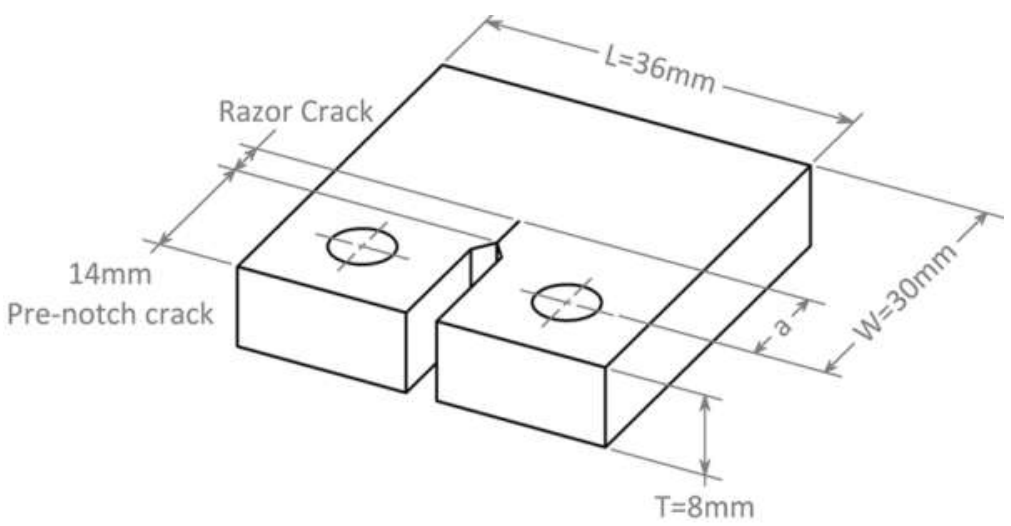

Figure 7.1. Geometry of compact tension specimen 
$K_{I C}=\frac{P \cdot F(x)}{T \cdot W}$

where $\mathrm{P}$ is the load applied, $\mathrm{T}, \mathrm{W}$ are the thickness and the width of specimens. $\mathrm{F}(\mathrm{x})$ is a function of $x=\frac{a}{W}$ and is defined as follows.

$F(x)=\frac{(2+x) \cdot\left(0.866+4.64 x-13.32 x^{2}+14.72 x^{3}-5.6 x^{4}\right)}{(1-x)^{3 / 2}}$

The plane strain energy release rate $\left(\mathrm{G}_{\mathrm{IC}}\right)$ can be calculated as follows,

$\mathrm{G}_{\mathrm{IC}}=\frac{\left(1-v^{2}\right) \cdot K_{I C}^{2}}{E}$

E: Young's modulus

$v$ Poisson's ratio

\subsubsection{Radiation attenuation performance}

The radiation attenuation testing was conducted at Royal Perth Hospital, Perth, Australiabased on the DIN 68571 testing method. The specimens $(30 \times 30 \mathrm{~mm})$ with a constant thickness of $8 \mathrm{~mm}$ and $16 \mathrm{~mm}$ at $\phi_{s}$ ranging from 0 to 0.14 were tested for X-ray attenuation. Each specimen was placed above an X-ray beam analyzer unit (Unfors Xi R/F \& MAM) at a fixed standard distance from the X-ray source. Attenuation was defined as the proportion of the primarybeam (from a GE Optima XR220AMX) absorbed by the specimen. Xray beam energies ranging from 60 to $120 \mathrm{kV}$ pwereused in the measurements. The X-ray protective capacity, A, was used to quantify the performance of the composite. This parameter is defined by

$A\left(\phi_{S}\right)=\frac{K(0)-K\left(\phi_{S}\right)}{K(0)}$

The air kerma (kinetic energy released per unit mass of air) $\mathrm{K}(\mathrm{Gy})$ is a quantitative parameter designed for radiation evaluation. $\mathrm{K}(0)$ and $\mathrm{K}\left(\phi_{s}\right)$ are the air kerma collected from an analyser unit when it is un-shielded and shielded by specimens at different particle volume $\operatorname{fractions}\left(\phi_{s}\right)$.

\subsection{Results and discussion}

\subsubsection{Morphology and chemical characterization of encapsulated nanofiller}


The particle size distribution result obtained with the Mastersizer shows a 13-fold decrease in medial size from $6 \mu \mathrm{m}$ of pristine to $0.45 \mu \mathrm{m}$ synthesized or treated (milled and SDS treated) particles. Likewise, the cumulative volume at $90 \%$ for the synthesized particles occurs at a size of less than $1.8 \mu \mathrm{m}$ compared to $30 \mu \mathrm{m}$ for the precursor. The SEM images of the precursor and treated particles shown in Figure 7.2A, Bindicate a very distinct morphology difference between the two types of powder. The precursor particles (Figure 7.2A) display porous near-spherical shaped particles with needle-like protrusions forming the surface. In contrast, the morphology of synthesized or treated particles has a rod-like shape (Figure 7.2B). A large amount of synthesized separated rod-like particles with a length of 1$2 \mu \mathrm{m}$ and a diameter of $50-80 \mathrm{~nm}$ were observed. The grinding and surface coating process transformed the bulky pristine $\mathrm{Gd}$ with a size greater than $10 \mu \mathrm{m}$ into nanorod particles.
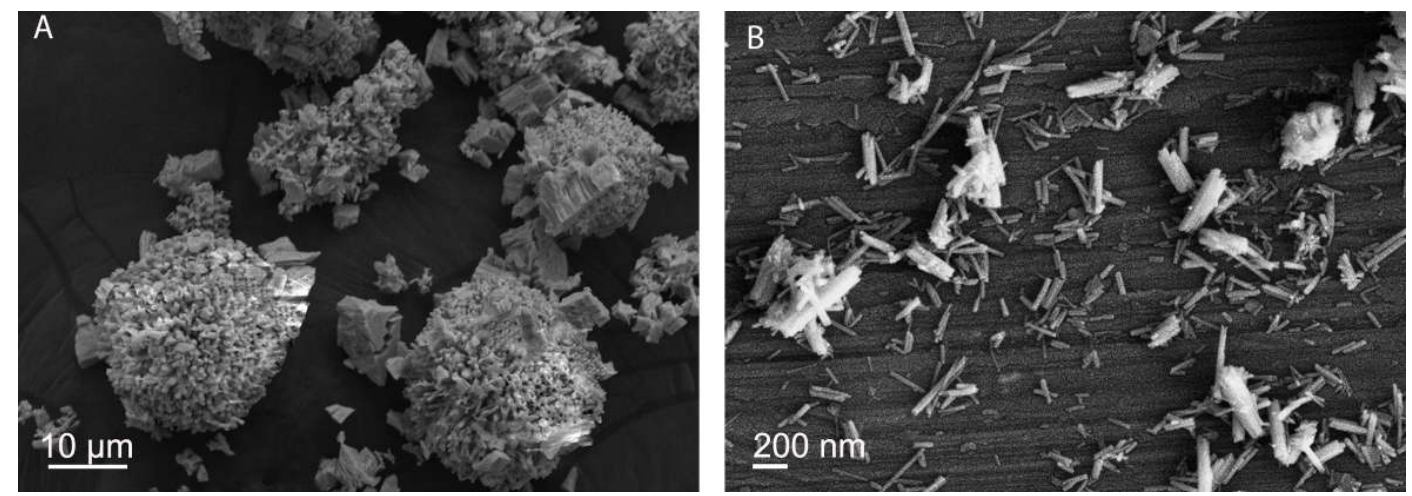

Figure 7.2. SEM morphologies of $\mathrm{Gd}_{2} \mathrm{O}_{3}$ at: (A) unmilled, (B) milled and SDS conditions.

Dimeric SDS molecules with two head-groups located at the opposite end of the hydrophobic bonded molecules were postulated to adsorb on the particle providing charge and steric stabilization. The interparticle repulsive force arising from the charged adsorbed surfactant layers is stronger than the particle van der Waal interaction, thereby causing particle segregation or dispersion. Therefore, this organic shell can prevent the coagulation of discrete ultrafine particles occurring during the sodium chloride washing and drying process (Figure 7.2) [207,208].

This work combines a high-yield, inexpensive grinding, and surface modification method to effectively create a hybrid core-shell nanoparticle for particulate composite fabrication using an ex-situ method, which is different to methods previously reported in the literature [7,57,170]. Furthermore, the capping agent also acts as(i) a steric barrier preventing the agglomeration or re-welding of the powder during nanoparticle preparation [2,94,166,208] and (ii) an intermediate adhesive that enhances the miscibility of the particle and liquid 
matrix, therebyimproving the particle dispersion in the organic matrix $[5,7,82,208]$. Other than physical anchoring [7,20], the strong ionic bond formed by electric charge adsorption between particle surface and surfactant enhances the particulate volume fraction limit for dispersion in the matrix, thereby improving the performance of final composite $[7,20]$. The preparation methodology developed in this study simplifies and purifies the ex-situ method of nanocomposite preparation by not using intermediate inert, volatile, and low viscous solvents such as tetrahydrofuran (THF), acetone, or ethanol to enhance the distribution of discrete fillers in the polymer matrix $[7,14]$.

The dried synthesized powder was characterized by FTIR to determine the presence of the organic shell encapsulating the Gd core. The FTIR spectra of Gd with and without the organic shell are shown in Figure 7.3. The spectra showed the presence of absorption bands for $\mathrm{Gd}_{2} \mathrm{O}_{3}$ at around $520 \mathrm{~cm}^{-1}$ and $1380 \mathrm{~cm}^{-1}$ for Gd-O stretching, and $1510 \mathrm{~cm}^{-1}$ for $\mathrm{Gd}=\mathrm{O}$ bending vibrations [7,211]. The appearance of aliphatic C-H stretching bands confirms the presence of an organic coating layer with 2 asymmetric and 1 symmetric stretching vibration in the region of $3000-2850 \mathrm{~cm}^{-1}$. Additionally, the peaks at around $1055 \mathrm{~cm}^{-1}$ and $693 \mathrm{~cm}^{-1}$ assigned to the asymmetrical stretching vibration of $\mathrm{S}=\mathrm{O}$ and $\mathrm{S}-\mathrm{O}$ respectively characterize the presence of SDS surfactant on the surface of the nanoparticle [212,213].

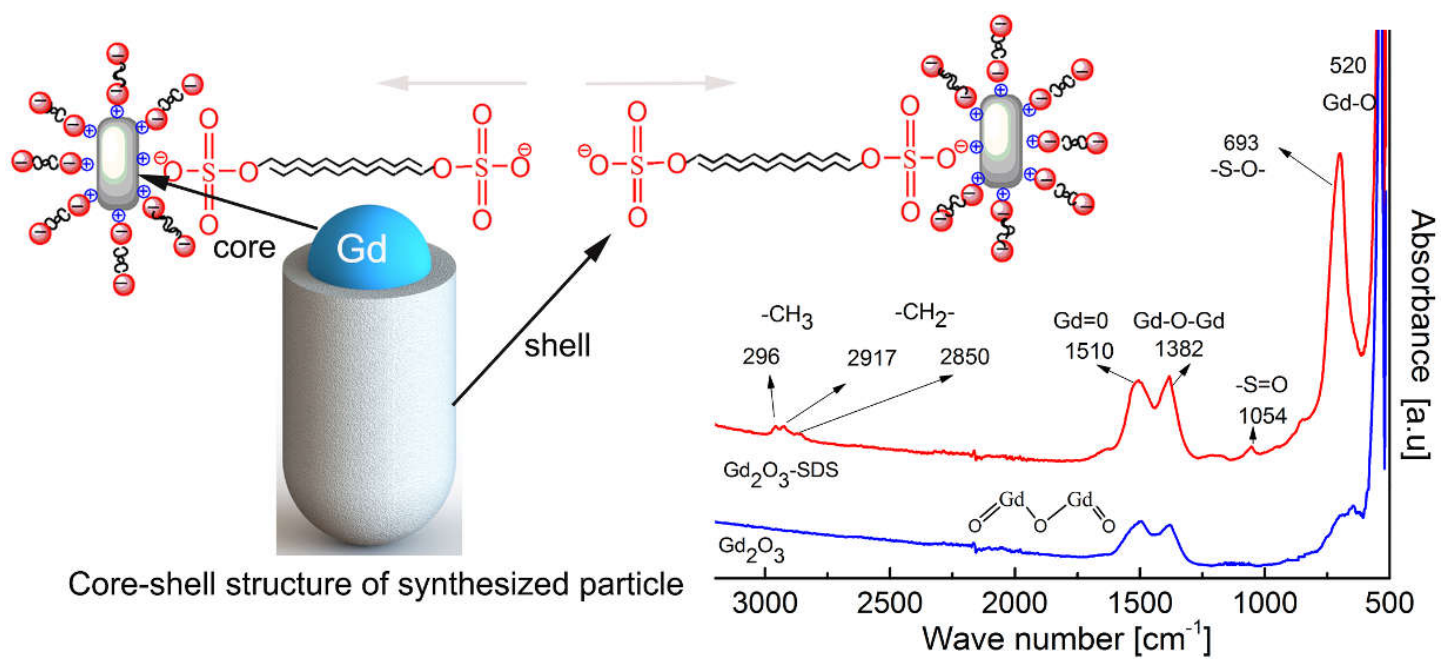

Figure 7.3. Schematic illustration of Gd nanoparticles surrounded by strongly adsorbed layer of organic molecules (core shell $\mathrm{Gd}_{2} \mathrm{O}_{3}$ particles) and their FTIR spectra.

\subsubsection{Mechanical properties and fractography of particulate composites}

Clear thermoset plastic formed by the cross linking reaction between prepolymer, bisphenol-A-(epichlorhydrin) (average molecular weight $\leq 700$ ) of low viscosity, and 
triethylenetetraminehardener was employed as a matrix for the dispersion of synthesized or treated Gd fillers. The mechanical properties of these composites were characterized to determine the limit of $\mathrm{Gd}$ particle load-carrying capacity in the epoxy, and also investigate the effectiveness of the particle surface treatment or dispersion. For reinforced epoxy with rigid fillers, the particle rigidity tends to strengthen the resultant polymer composite. The relationship between the fracture toughness of composite materials and the interfacial bonding between the nanoparticles and the polymer affecting the particle loading limit have been reported previously[217,218].

Figure 7.4A shows a decrease in tensile strength with particle volume fraction from 0 to 0.14. However, the Young's modulus increases with particle volume fraction and reaches a maximum value at 0.08 and then decreases again at higher volume concentration as seen in Figure 7.4B. The tensile strength of the materials decreases dramatically from $40 \mathrm{MPa}$ of neat epoxy to less than $10 \mathrm{MPa}$ of $\mathrm{Gd} /$ epoxy composite at $\phi_{s}=0.14$. The reduction in the tensile strength with particle volume fraction is as much as $30 \%$ from 0.04 to 0.06 . It is as much as $60 \%$ from 0.10 to 0.12 . Meanwhile, a roughly asymmetric peak-shaped curve characterizes the relationship between modulus strength and the particle volume concentration. A maximum value of the modulus of $1.25 \mathrm{GPa}$ is observed at $\phi_{s}=0.08$. The increase in modulus is about $25 \%$ from $\phi_{s}=0$ to $\phi_{s}=0.08$, meaning the material has a greater resistance to elastic deformation under load. In contrast, a dramatic reduction of Young's modulus in the range of volume fractions greater than 0.10 indicates a weak interface due to the increase of particle agglomeration in the matrix
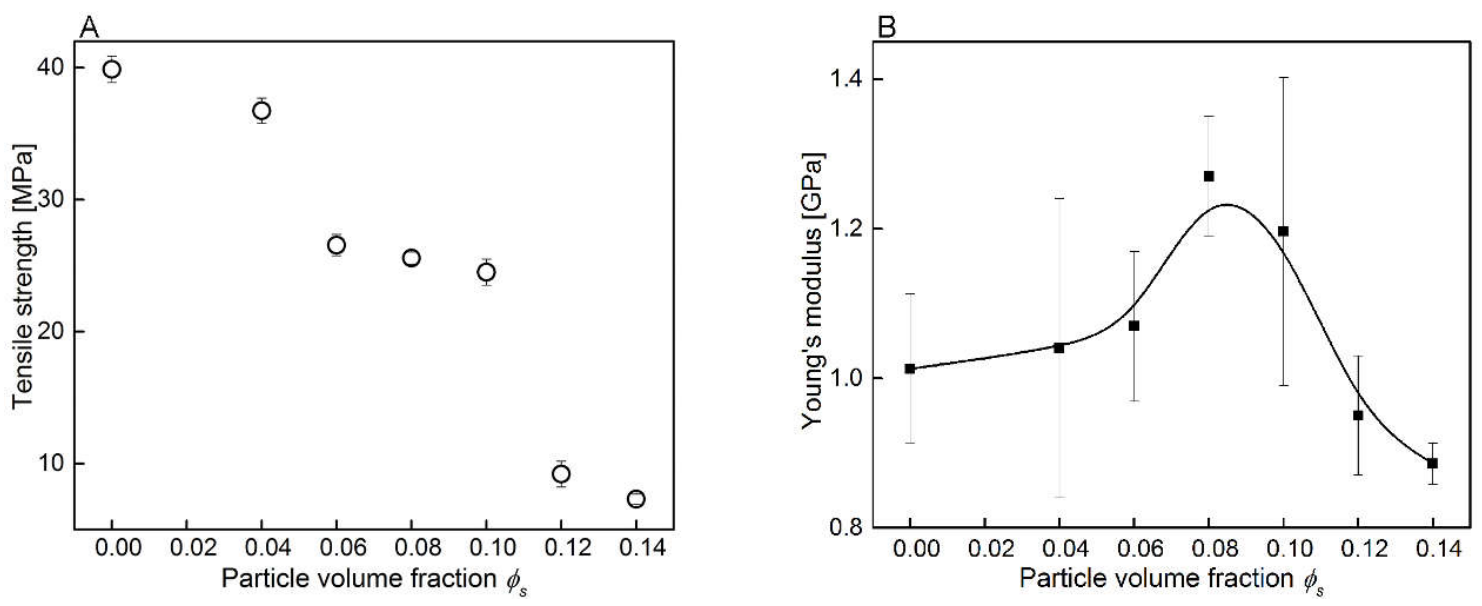

Figure 7.4. The relationship between (a) tensile strength and (b) Young's modulus with the volume fraction of $\mathrm{Gd}_{2} \mathrm{O}_{3}$ particles. 
Likewise, a peak in fracture toughness $\left(\mathrm{K}_{\mathrm{IC}}\right)$ is also displayed at particle volume fraction of 0.08 as shown in Figure 7.5A. The capacity of epoxy matrix containing a crack to resist fracture increases by up to $28-30 \%$ with the addition of $\mathrm{Gd}$ particles at particle volume fractions of 0.08 and 0.1 before exhibiting a dramatic decline at higher filler concentration. A similarly large increase in the energy release rate during fracture per unit $\left(\mathrm{G}_{\mathrm{IC}}\right)$ of $22-24 \%$ is observed over the same range of volumetric concentration of 0.08 to 0.1 . However, no significant reduction in this energy is observed at higher particulate concentrations.
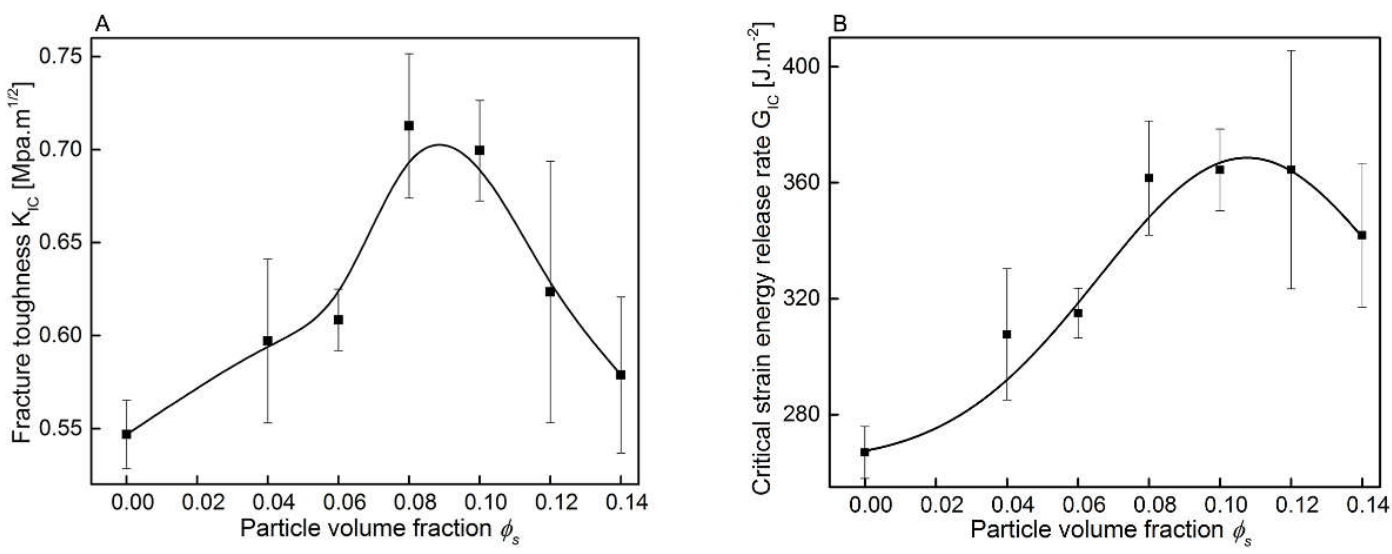

Figure 7.5. The fracture toughness of neat epoxy and its nanocomposites at different volume

\section{fractions}

A smooth fracture surface structure of the pure epoxy matrix is shown in Figure7.6A as this material is homogeneous. The SEM fracture surface is quite different in the presence of particles at $\phi_{s}=0.08$, as shown at different magnifications in Figure 7.6 B-E.The crack lines seen in $7.6 \mathrm{~B}$ extend in different directions, probably arising from microcracks formed at the strongly bonded interface of particle and matrix. This is a plausible reason for the increase in absorbed energy or fracture toughness[222-225]. Big clusters or aggregates usually reduce the impact strength because of the ease of major crack breakthrough as no voids or microcracks are found around these clusters (white circle in Figure 7.6D). In contrast, a single dispersion of Gd particles (as indicated by the red circles inFigure 7.6E) from good mixing extends the crack along the boundary surface of particle to create voids and microcracks when it encounters particles (Figure 7.7). In general, the level of plastic deformation is characterized by the width of the void, reflecting the effectiveness of interactions at the interface of the two phases and at high fracture toughness[218]. As seen from the facture morphologies shown in Figure 7.6B-E, there are relatively few agglomerates (or rough cracks) less than $2 \mu \mathrm{m}$ in sizeobserved on the fracture surface of the specimen. This observation impliesthe production 
of a well dispersed composite. The high concentration of voids or deformations in the epoxy matrix around the particle is also a reflection of the large amount of individual particles being well dispersed in the matrix. Additionally, the particle loading limit shows a $400-500 \%$ increment of particle volume fraction in epoxy matrix from 0.017 to $0.08-0.1$ compared to previous research [7]. This proves that the SDS surfactant improves the wettability and the interaction strength between particles and the matrix[7].
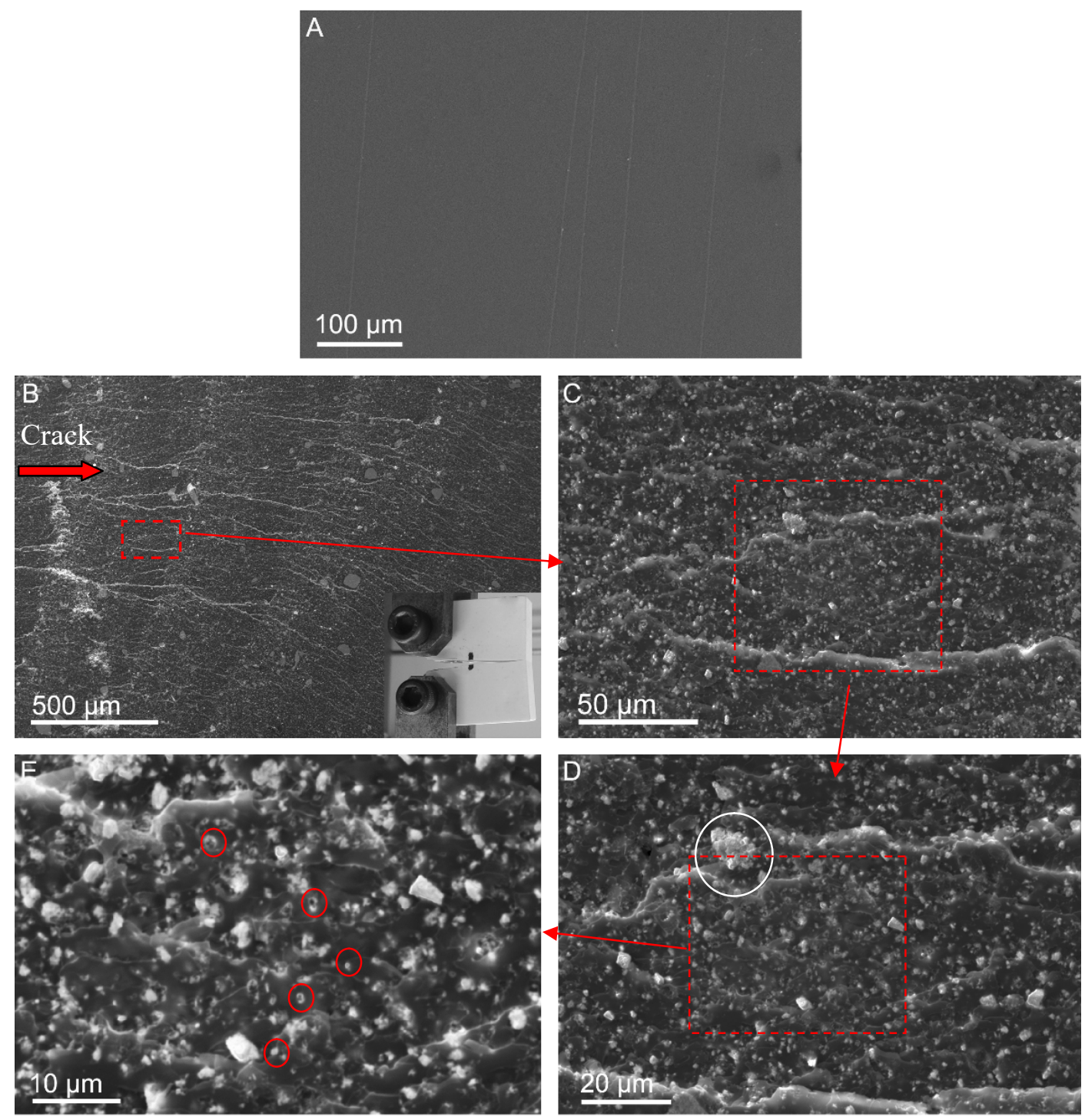

Figure 7.6. Fractograph of (A) neat epoxy matrix and (B, $C, D, E) G d$ nanoparticle $\left(\phi_{s}=0.08\right)$ epoxy composite at different magnifications.

Normally, there are two ways for a propagated crack to pass a particle, i.e. moving along particle-matrix boundaries and moving through the particle (fracturing the particle), as shown in Figure 7.7. The first route is the most common as the individual rigid particles are usually 
much stronger than the epoxy matrix, allowing particles to play a role as pinning obstructions of major cracks which need a greater force applied to continue propagating[226]. In this work, monodispersed Gd particles in matrix (Figure 7.7A), the crack growth deflects along particle boundaries, forms voids, then extends in different directions as microcracks. Debonding and matrix deformation at the particle-matrix interface requires external stretching load or energy to form local elastic cavities around particles, or blank voids from particle pull-out and microcracks around particles [227]. The extension and development of the cracksof the filled polymer material increases the total propagated crack length and hence requires a higher strain load or absorbs more energy than unfilled one [228]. Strong chemical bonding between particles and the matrix through an adhesive layer also consumes more energy for debonding and matrix deformation around the particles [218]. This mechanism explains the increased plane strain energy release rate $\left(\Delta \mathrm{G}_{\mathrm{IC}}\right)$ of Gd-filled composite compared with unfilled polymer material. In contrast, a propagating crack passes through bulky agglomeration under very low strain load from a fracture toughness test because of low interparticle attractive force, so no energy is absorbed (Figure 7.7B). Excessive particle density per unit volume increases the tendency of agglomeration, thereby reducing the fracture toughness of theGd-filled composite [229].
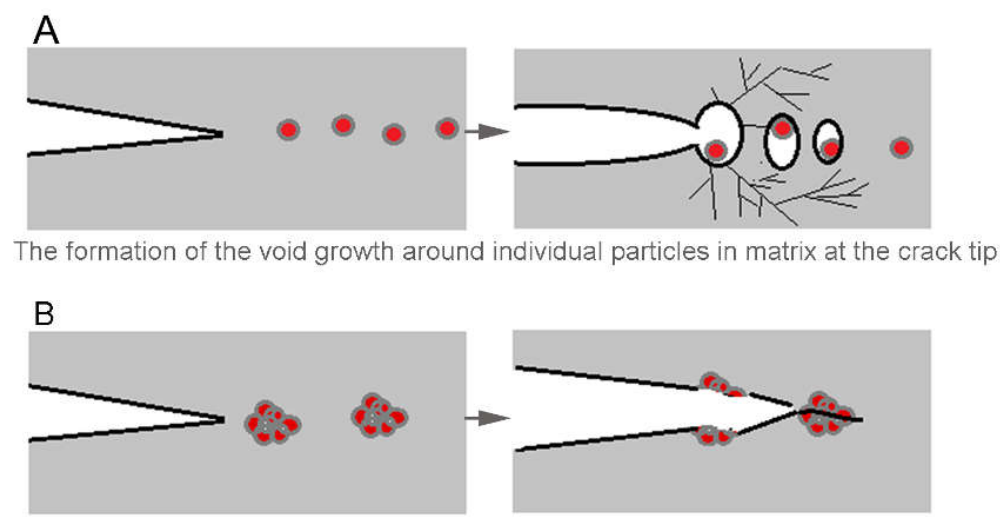

The growth of crack tip through the large particle clusters in matrix

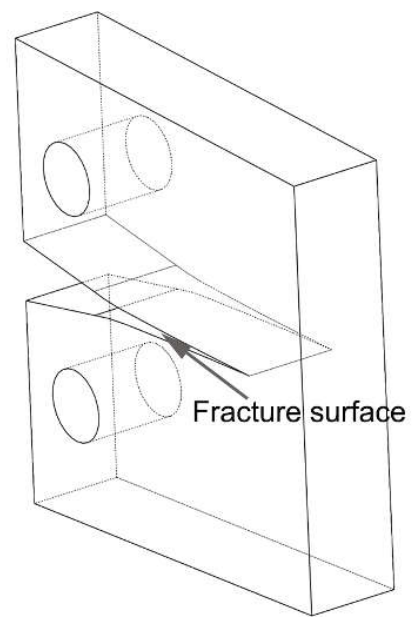

Figure 7.7. Mechanism of crack growth paths for organic encapsulated nano $\mathrm{Gd}_{2} \mathrm{O}_{3}$ particles in epoxy resin.

\subsubsection{X-ray attenuation characterization}

The relationship between solid volume fractions of synthesized nanoparticles in epoxy at thicknesses of $8 \mathrm{~mm}$ and $16 \mathrm{~mm}$, and X-ray attenuation is determined in this research. To investigate the shielding properties of the $\mathrm{Gd}_{2} \mathrm{O}_{3}$-epoxy samples developed in this work, 
seven beam energies of $60,70,80,90,100,120 \mathrm{kVp}$ are selected for the attenuation test.

Figure 7.8shows an increase in attenuation with particle volume fraction. The relationship between attenuation coefficient $(A)$ and a volume fraction $\left(\phi_{s}\right)$ all fitan exponential association model at different levels of photon energy described by the equation below:

$A=a_{1} \cdot\left(1-e^{-\frac{\phi_{s}}{b_{1}}}\right)+a_{2} \cdot\left(1-e^{-\frac{\phi_{s}}{b_{2}}}\right)+c$, with $\left(0 \leq \phi_{\mathrm{s}} \leq 1\right)$ and $(0 \% \leq \mathrm{A} \leq 100 \%)(7.7)$

where $\mathrm{a}_{1}, \mathrm{a}_{2}, \mathrm{~b}_{1}, \mathrm{~b}_{2}$ are constants and $\mathrm{c}$ is the $A$-intercept of $A$ - $\phi_{s}$ regression. Radiation protective effectiveness initially increases rapidly with an addition of Gd particles at volume fraction of 0.04 . This increase is $76-86 \%$ ( $8 \mathrm{~mm}$ thickness) and $92-96 \%$ ( $1 \mathrm{~mm}$ thickness) in the range energy of $120-60 \mathrm{kVp}$. After that, X-ray protective performance increases steadily with increased solid volume fraction. An $8 \mathrm{~mm}$ thick $\mathrm{Gd}_{2} \mathrm{O}_{3}$-filled composite slabin the volume fraction range of 0.04 to 0.14 attenuates more than $76 \%$ of a $120 \mathrm{kVp}$ primary X-ray beam; doubling the slab thickness increases the attenuation fraction to $92 \%$. As expected, there is a relationship between the thickness of the composite and the X-ray attenuation efficiency. X-ray attenuation also varies substantially with beam energy. The relationship between radiation attenuation and beam energy are described inFigure
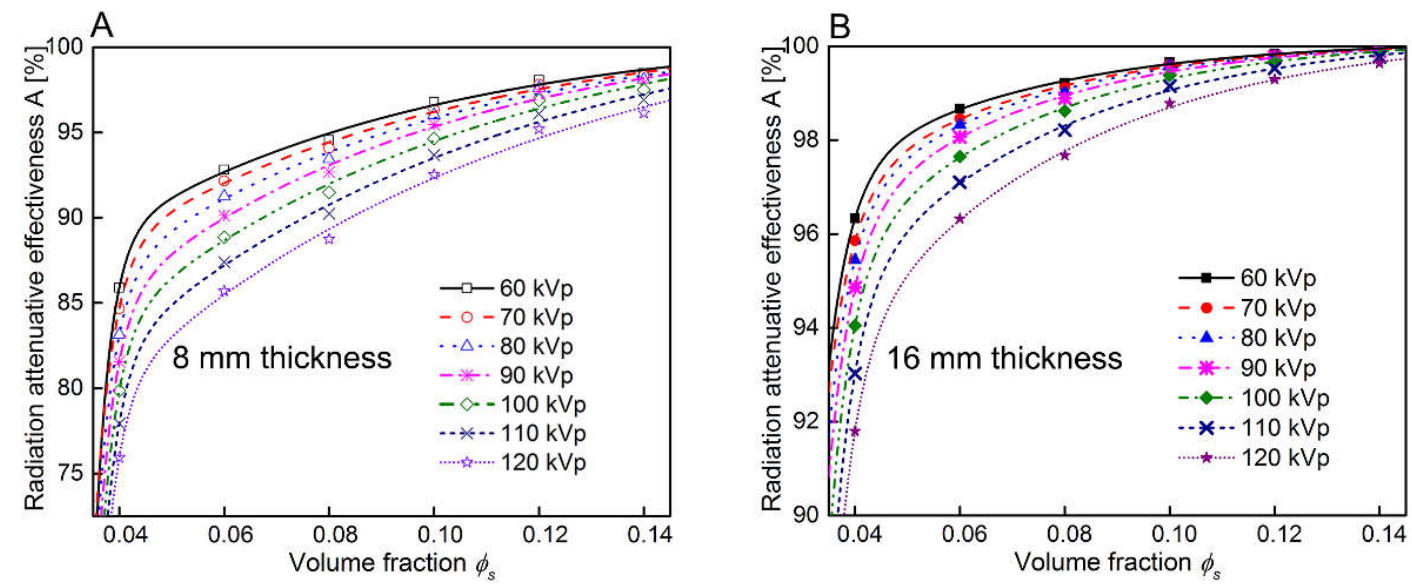

Figure 7.8. The relationship between X-ray attenuation coefficient (A) and volume fraction $\left(\phi_{s}\right)$ for the Gd-filled composites with (a) $8 \mathrm{~mm}$ and (b) $16 \mathrm{~mm}$ thicknesses.

Figure 7.9shows that the relationship between X-ray attenuation and beam energy (E) at different volume fractions $\left(\phi_{s}\right)$ follows a mathematical of exponential decay modal as follows: $A=a_{3} \cdot e^{-\frac{E}{b}}+b_{3}$

where $\mathrm{E}(\mathrm{kVp})$ is beam energy, $\mathrm{a}_{3}$ is a constant, and $\mathrm{b}_{3}$ is the attenuation coefficient $(A)$ 
intercept of regression

As shown from the dependence of X-ray attenuation on the thickness of the material and volume concentration of $\mathrm{Gd}_{2} \mathrm{O}_{3}$ (Figure 7.8 and 7.9), the attenuation performance increased with both the thickness and particulate concentration. Normally, the effect of particle volume fraction isconsidered first from the perspective of the product weight. A product would ideally utilize the lowest weight per unit volume of composite that provides adequate shielding.An excessive increase in the discrete phase would add unnecessary weight and significantly increase the likelihoodof mechanical failure. The ideal particle volume fraction range is determined in this work to be 0.08 to 0.1 due to significant improvement of mechanical properties observed in this range. If greater attenuation is required, the composite thickness can be increased.

The next step will investigate the dependence of the degree of X-ray attenuation on the composite thickness and also do an evaluation on X-ray attenuation efficiency/weightthickness (or weight per unit area) of the material. To find an optimal balance between fracture toughness properties and functional radiation attenuation, the particle loading volume fractions of 0.08 and 0.1 or density in the range of $0.171-0.183 \mathrm{~g} / \mathrm{cm}^{3}$ of composite with a thickness of $8 \mathrm{~mm}$ and $16 \mathrm{~mm}$ respectively are used. The weight/radiation attenuation proficiency evaluation required that the particulate volume fractions of the composite be converted to weight per unit area $\left(\mathrm{g} / \mathrm{cm}^{2}\right)$
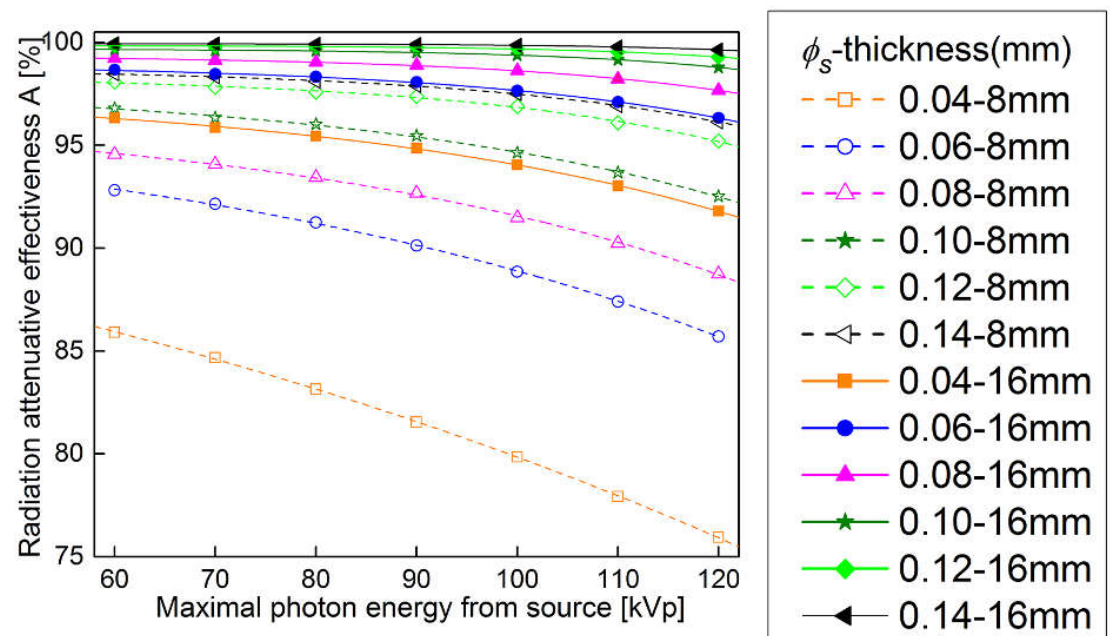

Figure 7.9. The relationship between X-ray attenuation and beam energy (E) at different volume fraction $\left(\phi_{s}\right)$

Normally, the X-ray protective performance is evaluated in terms of the lead thickness 
required to provide the same shielding effect (lead equivalent). Depending upon the environment of application, different $\mathrm{Pb}$ equivalent shields are required. $\mathrm{Pb}$ equivalences of $0.25,0.35$, or $0.5 \mathrm{~mm}$ are required in protective aprons in various clinical environments [53]. $\mathrm{A} \mathrm{Pb}$ equivalence of $1 \mathrm{~mm}$ is often used in portableshieldingin clinical radiology[53]. The density of lead, and therefore the weight of lead-based shields, is one of the major factors driving the development of alternative shielding materials.

Figure 7.10A shows that the performance of the X-ray attenuation of the $8 \mathrm{~mm}$ composite with $\phi_{s}$ of 0.08 is equivalent to $0.2 \mathrm{~mm} \mathrm{~Pb}$, and the $16 \mathrm{~mm}$ composite being equivalent to 0.5 $\mathrm{mm} \mathrm{Pb}(\mathrm{A}>95 \%)$. Likewise, $8 \mathrm{~mm}$ and $16 \mathrm{~mm}$ sheets with $\phi_{s}$ of 0.1 produce radiation attenuation performance equivalent to $0.35 \mathrm{~mm} \mathrm{~Pb}(\mathrm{~A}>90 \%)$ and $1 \mathrm{~mm} \mathrm{~Pb}(\mathrm{~A}>99 \%)$ respectively $[10,11,53]$. At the higher energy range $(\mathrm{kVp}>80)$ the composite actually performs better that its $\mathrm{Pb}$ equivalent sheet. The weight-thickness of composites with a high X-ray protection equivalent to 0.5 and $1 \mathrm{~mm} \mathrm{~Pb}$ is $16 \mathrm{~mm}$ thick with $\phi_{s}$ of $0.08(\mathrm{~d}=$ $\left.1.581 \mathrm{~g} / \mathrm{cm}^{3}\right)$ and $0.10\left(\mathrm{~d}=1.706 \mathrm{~g} / \mathrm{cm}^{3}\right)$ are compared with other convenient materials such as wood, glass, concrete, and especially commercial non-lead X-ray attenuating materials (Figure 7.10B).

A comparison of weight per area unit between Gd particulate epoxy and other materials at the same shielding performance is made. $1 \mathrm{~mm} \mathrm{~Pb}$ equivalent wood, glass, and concrete are nearly $16,8.5$ and 7 times heavier than that of $\mathrm{Gd}_{2} \mathrm{O}_{3}$ specimens, respectively $[53,55,69]$. Although the weight per unit area $\left(\mathrm{g} / \mathrm{mm}^{2}\right)$ of $\mathrm{Pb}$ sheet is $40-80 \%$ less than the $\mathrm{Gd}_{2} \mathrm{O}_{3}$ sample in the range $60-120 \mathrm{kVp}$, pure $\mathrm{Pb}$ is toxic and hence is restricted for use as personal protective equipment. $\mathrm{Pb}$ impregnated polymer is used instead, but this product possesses inferior radiation shielding qualities and weighs more than pure $\mathrm{Pb}[10,53,191]$. Although the use of toxic $\mathrm{Pb}$-based materials is more cost effective, nowadays non-lead barriers are becoming more common to satisfy safe use, manufacturing, and disposal regulations [10,11]. The weight per area unit of $16 \mathrm{~mm}$ thickGd composite with $\mathrm{d}=1.581 \mathrm{~g} / \mathrm{cm}^{3}\left(\phi_{s}=0.08\right)$ at $0.5 \mathrm{~mm}$ $\mathrm{Pb}$ attenuation equivalence compares favourably in X-ray protective performance with other commercial non-lead based materials.Aprons from various suppliersincluding Xenolite and EarthSafe all demonstrate a weight-thickness per square millimeter value that is $20-40 \%$ less than commercial $\mathrm{Pb}$ based material [11]. The composite developed in this work has a 4.5$19.4 \%$ lower weight per unit area than Xenolite and EarthSafe products at $0.5 \mathrm{~mm} \mathrm{~Pb}$ equivalence. Additionally, the Gd-composite out-performed a $0.5 \mathrm{~mm}$ lead equivalence and several non-lead products in the $75-120 \mathrm{kVp}$ energy range (Figure $7.10 \mathrm{~A}$ ). Regarding the 
radiation protective performance per unit weight, $\mathrm{Gd}_{2} \mathrm{O}_{3}$-content shields are a reasonable alternative for $\mathrm{Pb}$ and even non- $\mathrm{Pb}$ based materials for diagnostic $\mathrm{X}$-ray beams up to $120 \mathrm{kVp}$.
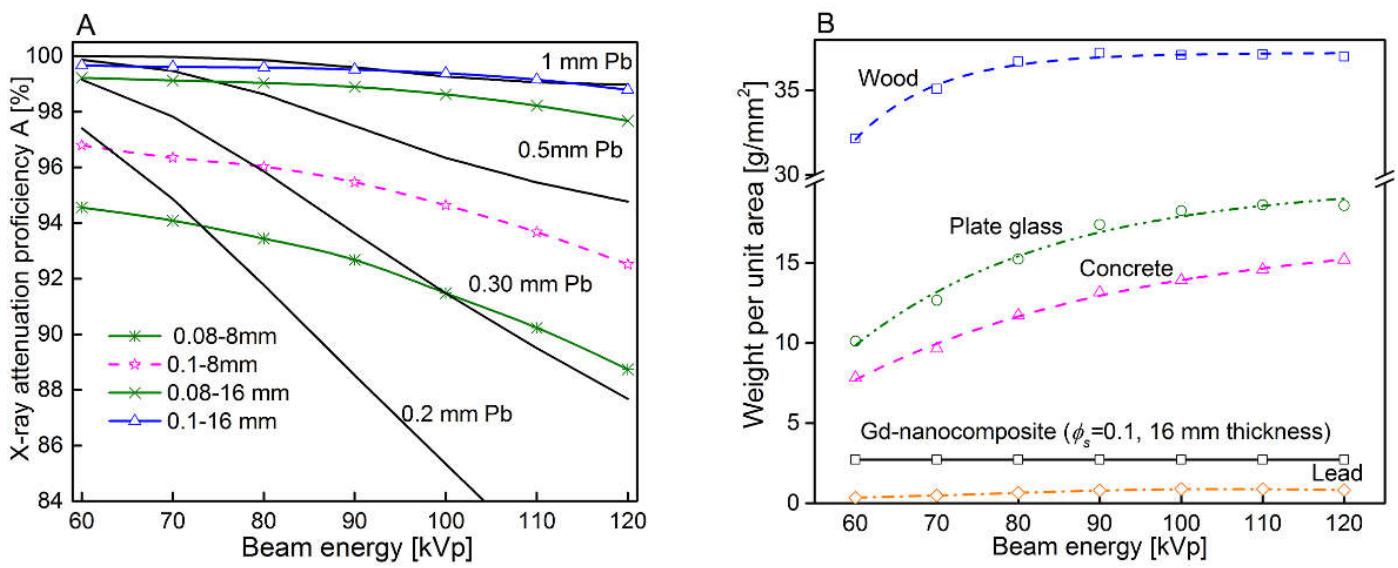

Figure 7.10. (A) The effective radiation attenuation of particulate composite $\left(\phi_{s}=0.08\right.$, $\left.\phi_{s}=0.1\right)$ at different thicknesses and lead equivalences, and (B) the comparison of weight per unit area at the same radiation attenuation proficiency between $\mathrm{Gd}_{2} \mathrm{O}_{3}$ /epoxy composite ( $\phi_{s}$ $=0.1,16 \mathrm{~mm}$ thickness) and some convenient materials sheeting across a range of diagnostic beam energies

\subsection{Conclusion}

An inexpensive and effective process to produce a lead-free material for diagnostic energy X-ray protectionis presented. Additionally, the correlation between the dispersion status, the functional properties and mechanical performance of the composite obtained in this investigation also provide new knowledge.Surface modification of the particles with SDS facilitates both the nanoparticle preparation and dispersion in epoxy. The use of inexpensive rough $\mathrm{Gd}_{2} \mathrm{O}_{3}$ particle as starting material, followed by milling and surface modification, is a simple method for manufacturing well dispersed nanoparticulate-epoxy composite. A composite sheet containing 0.08 to 0.1 volume fraction of synthesized core-shell $\mathrm{Gd}$, dispersed well in an epoxy matrix with a thickness of 8 or $16 \mathrm{~mm}$, can satisfy all levels of Xray protection required in the clinic, while having a reasonable weight per unit area. The nanoparticulate fillers also increase the fracture toughness properties of the epoxy by $28-30 \%$. This research successfully providesa design and preparation process of a new "green" material for radiation shielding. The compatibility of the particle and epoxy is an important factor affecting the mechanical and functional performance of the composite, as it determines the microstructure of the composite. 